\title{
EFFECT OF VAGINAL SILDENAFIL ON IN VITRO FERTILIZATION SUCCESS RATES IN WOMEN WITH PREVIOUS FAILED IN VITRO FERTILIZATION ATTEMPTS
}

\author{
ENSIEH SHAHROKH TEHRANINEJAD ${ }^{1}$, NOUSHIN KHAZEI ${ }^{2}$, ELNAZ AYATI ${ }^{1}$, ALI MOVAFEGH ${ }^{2}$, OMID AZIMARAGHI ${ }^{2 *}$ \\ ${ }^{1}$ Department of Obstetrics and Gynecology, Tehran University of Medical Sciences, Iran. ${ }^{2}$ Department of Anesthesiology and Critical Care, \\ Tehran University of Medical Sciences, Iran. Email: o.azimaraghi@gmail.com
}

Received: 05 March 2018, Revised and Accepted: 04 April 2018

\section{ABSTRACT}

Objectives: Endometrial thickness of $<9 \mathrm{~mm}$ is a predictor of in vitro fertilization (IVF) failure, although neither pregnancy rates nor the pregnancy outcomes are dependent on the endometrial thickness alone. The impact that uterine artery blood flow has on endometrial growth is dependent on nitric oxide which concentrations could be altered by halting a cyclic guanosine monophosphate-mediated pathway with a phosphodiesterase type 5 selective inhibitor such as sildenafil.

Methods: In this clinical trial, 72 patients aged below 45 years which have had at least two earlier failed IVF attempts were randomly split into two groups each consisting of 36 patients. Both groups were started on a long IVF protocol. The case group was also administered 100 mg vaginal sildenafil suppositories daily, starting on day 3 of menstruation which was continued until human chorionic gonadotropin administration. Endometrial thickness was measured using ultrasonography in both groups plus pregnancy rates were assessed in both groups.

Results: The mean age of the patients in Group A who received sildenafil was 33.8 \pm 4.8 in contrast to Group B (control group) with the mean age of 33.8 \pm 4.8 . Mean endometrial thickness of $8.6 \pm 0.1 \mathrm{~mm}$ was recorded in Group B compared to $9.0 \pm 0.7 \mathrm{~mm}$ in Group A ( $\mathrm{p}=0.03$ ). Of all the 36 participants who received sildenafil citrate during the IVF cycle, 12 (33.3\%) patients had successful pregnancies while 24 (66.7\%) failed to get pregnant. In the control group, out of the 36 participants, 10 (27.8\%) patients got pregnant while $26(72.2 \%)$ failed the cycle (p=0.9).

Conclusion: This study showed that although using vaginal sildenafil during the IVF cycle does improve endometrial thickness before implantation, this does not necessarily lead to higher pregnancy rates.

Keywords: Infertility, Sildenafil, In vitro fertilization.

(C) 2018 The Authors. Published by Innovare Academic Sciences Pvt Ltd. This is an open access article under the CC BY license (http://creativecommons. org/licenses/by/4. 0/) DOI: http://dx.doi.org/10.22159/ajpcr.2018.v11i6.25645

\section{INTRODUCTION}

Infertility is defined as incapability to conceive despite having regular unprotected sex for at least a year [1,2]. Around one in six or seven couples may have difficulty getting pregnant. Unexplained infertility in addition to common causes of infertility such as ovulation problems, tubal blockage, male associated infertility, uterine problems, and infections are causes which could eventually lead to using associated reproductive technology commonly known as ART in the treatment of infertility [3-6]. In vitro fertilization (IVF) as a helpful way to treat infertility, particularly fallopian tube and ovarian-related infertility, may lead to failure due to many factors such as female age, embryo quality, ovarian response, or implantation issues. Endometrial thickness of $<9 \mathrm{~mm}$ has been long known to be a predictor of IVF failure, although neither pregnancy rates nor the pregnancy outcome is dependent on the endometrial thickness alone [7]. A thin endometrial lining may mirror previous or a current uterine pathology, and with the importance that uterine artery blood flow has on endometrial growth, we could conclude that improving uterine blood flow may facilitate achieving higher pregnancy rates. The significance of nitric oxide (NO) as a modulator of uterine blood flow through the relaxation of vascular smooth muscle by a cyclic guanosine monophosphate (cGMP)mediated pathway has been studied [8]. Phosphodiesterase (PDE) enzymes also play a role in the NO-CGMP cycle by hydrolyzing cGMP through degrading the phosphodiester bond in these second messenger molecules and so regulating the signal transduction mediated by cGMP. PDE inhibitors (PDE I) can prevent this degrading of cGMP molecules by inhibiting the PDE enzyme.
Sildenafil (Viagra) is a PDE type 5 selective inhibitor, which facilitates the vasodilatory effects of cGMP in the corpus cavernosum and is used to treat erectile dysfunction, as well as having some other medical applications such as treatment of pulmonary hypertension $[9,10]$.

\section{METHODS}

Following approval of the Ethics Committee of Tehran University of Medical Sciences, with the aim of evaluating the effect of vaginal sildenafil suppositories on endometrial thickness and IVF success rate, a total number of 72 subjects were selected from the patients who were referred to Emam Hospital over a period of 7 months.

The following inclusion criteria were applied for selecting the patients eligible to take part in this study:

Women who had previously at least two IVF failure attempts and women aged below 45 years of age.

Patients are 45 years of age or older and patients with a history of heart failure (function class III or higher), signs of severe or chronic ischemia of the lower extremities, or pulselessness on physical examination, a previously diagnosed hematological, renal, hepatic or endocrinal disorder, medical history of acute/chronic heart disease, or high blood pressure were not included in the study.

The patients should also be mentally intact to be able to give a written form of consent. After the patients had been selected to enroll in the study, all participants were fully informed of the nature of the study 
and provided a written informed consent. In the primary assessment, a detailed medical history was taken which stressed on duration since the $1^{\text {st }}$ time diagnosis of infertility, previous IVF attempts, previous treatments used, and allergies to any drugs. The patients were randomly divided into two groups. In both groups, on day 21 of the previous cycle, patients were admitted to the hospital and were injected subcutaneously 3 times a day at $8 \mathrm{~h}$ intervals of $0.5 \mathrm{~mL}$ Suprefact (Category A: 21.10: Trophic hormones), for 7 days. Each $1 \mathrm{~mL}$ of the aqueous solution contains $1.05 \mathrm{mg}$ buserelin acetate corresponding to $1 \mathrm{mg}$ buserelin base as the active ingredient. On day 3 of menstrual cycle, Gonal-F was started in both groups. Daily $100 \mathrm{mg}$ vaginal sildenafil suppositories were also administered to the patients in the case group from the $3^{\text {rd }}$ day of menstruation which was continued until to the date of human chorionic gonadotropin (HCG) administration. Endometrial thickness in both groups was measured by a previously trained physician. All the patients were followed by clinical pregnancy or failure was recorded. Statistical analysis of the results was performed using SPSS for windows version 11.5, and data are presented as means \pm standard deviation. Independent paired t-test was performed for the comparison of the case group who received vaginal sildenafil and the control group. $\mathrm{p}<0.05$ was statistically considered significant.

\section{RESULTS}

\section{Basic characteristics}

A total number of 72 patients aged between 21 and 43 years who were compliant with the inclusion and exclusion criteria were enrolled in this study. The mean age of the patients in Group A who received sildenafil (case group) was $33.8 \pm 4.8$ in contrast to Group B (control group) with the mean age of $33.8 \pm 4.8$.

The mean duration of previously diagnosed infertility before treatment in Group A and B was 10.3 and 10.1 of years. The mean number of ova conceived during the IVF cycle in Group A was 9.6 \pm 5.9 , and in the control group, the mean number of ova's conceived was $9.3 \pm 6$.

Mean number of embryos transferred in the case group was $2.8 \pm 2.0$ in contrast to $2.8 \pm 0.8$ in the control group.

\section{Adverse drug effects}

During the IVF cycle, the patients were evaluated for any adverse drug reactions to sildenafil through physical examinations, but no clinically significant side effects were observed and all the patients tolerated the drug during the treatment.

\section{Endometrial thicknesses and pregnancy}

The 36 patients in Group B (control group) who did not receive the drug were undergone vaginal sonography with the mean endometrial thickness of $8.6 \pm 0.1 \mathrm{~mm}$. All the patients who were randomly chosen to be in Group A (case group) and received sildenafil suppositories were also undergone vaginal sonography. The mean endometrial thickness in Group A was $9.0 \pm 0.7 \mathrm{~mm}$ which were statistically significant compared to the endometrial thickness in Group B ( $p=0.03)$. Of all the 36 participants who received sildenafil citrate during their IVF cycle, 12 (33.3\%) patients had successful pregnancies while $24(66.7 \%)$ failed to get pregnant. In the control group, out of the 36 participants, $10(27.8 \%)$ patients got pregnant while $26(72.2 \%)$ failed the cycle $(p=0.9)$.

\section{DISCUSSION}

The fact that better endometrial thickness could help achieve higher pregnancy rates is understandable and has been made known [11], the hypothesis that vaginal sildenafil could directly increase clinical pregnancy rates, although previously studied has not been well established.

Vaginal sildenafil has been shown to have favorable effects on endometrial thickening as it is observed in previous studies $[12,13]$. In this study, we showed that using vaginal sildenafil during the IVF cycle does improve endometrial thickness, although there have been contraindicating results in other studies too.
Endometrial thickening is most probably the result of sildenafil acting as a PDE type 5 selective inhibitor, which helps accumulate NO through a cGMP pathway resulting in better blood flow.

Although higher concentrations of NO could result in better blood flow in the uterine, based on previous studies conducted on mice, it could also inhibit both embryo development in vitro and implantation in vivo [14], which is why discontinuing sildenafil administration before the date of HCG administration was proposed by Sher et al. [12]. It has also been proposed that the GMP pathway might be involved in the NOregulated embryonic development, but not in NO-induced apoptosis which could lead to errors during implantation.

Although no specific side effects or toxicity was observed in our study, with the fact that NO has important roles during embryo development and using sildenafil citrate during the IVF cycle, the NO concentrations are altered more studies are needed to verify the safety of vaginal sildenafil on the babies.

In cases where infertility is specifically related to a deficiency in blood flow such as Asherman's syndrome, treatment cycle with vaginal sildenafil citrate is effective [15]. In a retrospective cohort analysis of 105 infertile women aged $<40$ years, with at least two consecutive prior IVF failures attributed to inadequate endometrial development Sher et al. expressed that sildenafil citrate could enhance the endometrium development although they did not clearly evaluate the difference in the pregnancy rates [13].

From the results of this study, it may be concluded that although using vaginal sildenafil during the IVF cycle does improve endometrial thickness before implantation, this does not mean that higher pregnancy rates that are statistically significant will be achieved which highlights other roles that NO could have during the process apart from muscle relaxation and increase blood flow.

\section{AUTHOR'S CONTRIBUTION}

All the authors have contributed equally.

\section{CONFLICTS OF INTEREST}

The authors declare that they have no conflicts of interest.

\section{REFERENCES}

1. Hanson B, Johnstone E, Dorais J, Silver B, Peterson CM, Hotaling J. Female infertility, infertility-associated diagnoses, and comorbidities: A review. J Assisted Reprod Genet 2017;34:167-77.

2. Kukla R. Infertility, epistemic risk, and disease definitions. Synthese 2017:1-20.

3. Ramgir SS, Abilash VG. Genetic and environmental factors involved in human male infertility: A review. Asian J Pharm Clin Res 2015;8:34-43.

4. Anwar S, Anwar A. Infertility: A review on causes, treatment and management. Women's Health Gynecol 2016;2:2-5

5. Zoe R. Causes of infertility in women at reproductive age. Health Sci J 2009;3:80-7.

6. Sahu MC, Mishra SP, Panda R, Patnaik T. Surveillance of microbial flora for infertility couples in an indian tertiary care teaching hospital. Asian J Pharm Clin Res 2017;8:409-16.

7. Griesinger G, Trevisan S, Cometti B. Endometrial thickness on the day of embryo transfer is a poor predictor of IVF treatment outcome. Hum Reprod Open 2018;2018:hox031.

8. Alexander BT, Kassab SE, Miller MT, Abram SR, Reckelhoff JF, Bennett WA, et al. Reduced uterine perfusion pressure during pregnancy in the rat is associated with increases in arterial pressure and changes in renal nitric oxide. Hypertension 2001;37:1191-5.

9. Wareing M, Myers JE, O'hara M, Baker PN. Sildenafil citrate (Viagra) enhances vasodilatation in fetal growth restriction. J Clin Endocrinol Metab 2005;90:2550-5.

10. Unegbu C, Noje C, Coulson JD, Segal JB, Romer L. Pulmonary hypertension therapy and a systematic review of efficacy and safety of PDE-5 inhibitors. Pediatrics 2017;139:e20161450.

11. Holden EC, Dodge LE, Sneeringer R, Moragianni VA, Penzias AS, 
Hacker MR. Thicker endometrial linings are associated with better IVF outcomes: A cohort of 6331 women. Hum Fertil 2017:1-6.

12. Sher G, Fisch JD. Vaginal sildenafil (Viagra): A preliminary report of a novel method to improve uterine artery blood flow and endometrial development in patients undergoing IVF. Hum Reprod 2000;15:806-9.

13. Sher G, Fisch JD. Effect of vaginal sildenafil on the outcome of in vitro fertilization (IVF) after multiple IVF failures attributed to poor endometrial development. Fertil Steril 2002;78:1073-6.

14. Barroso RP, Osuamkpe C, Nagamani M, Yallampalli C. Nitric oxide inhibits development of embryos and implantation in mice. Mol Hum Reprod 1998;4:503-7.

15. Zinger M, Liu JH, Thomas MA. Successful use of vaginal sildenafil citrate in two infertility patients with Asherman's syndrome. J Women's Health 2006;15:442-4. 